\section{LA-UR-9 $3-32 \circlearrowleft 8$}

Title:

Author(s):

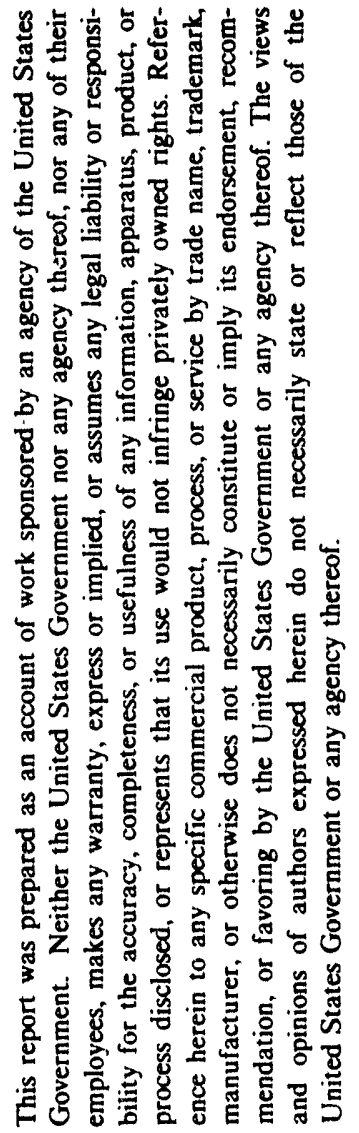

ORIENTATION IMAGING MICROSCOPY:

NEW POSSIBILITIES FOR MICROSTRUCTURAL INVESTIGATIONS USING AUTOMATED BKD ANALYSIS
Brent L. Adams, Brigham Young University David J. Dingley, Bristol University, UK Karsten Kunze, Brigham Young University Stuart I. Wright, MST-6
Submitted to:

International Conference on Textures of Materials Clausthal, Germany

September 20-24, 1993

\section{Los Alamos} NATIONAL LABORATORY
EIVED
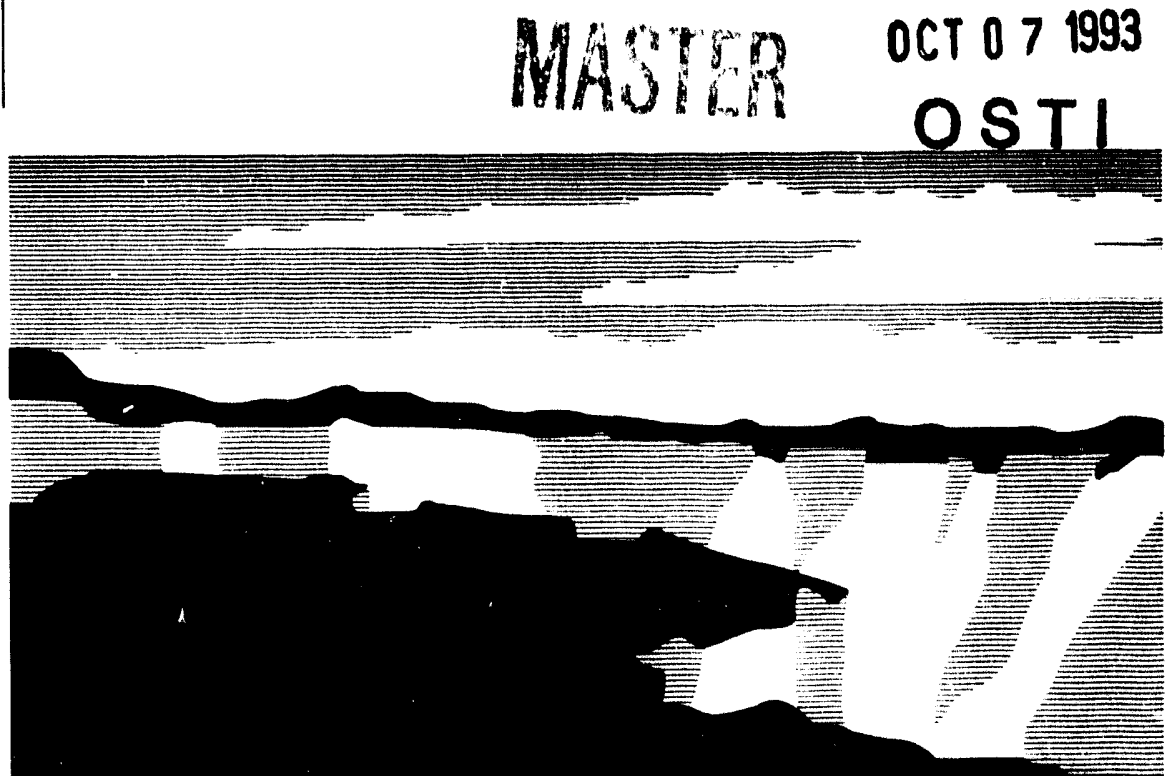

Los Alamos National Laboratory, an aftirmative action/equal opportunity employer, is operated by the University of California for the U.S. Department of Energy under contract W-7405-ENG-36. By acceptance of this article, the publisher recognizes that the U.S. Government retains a nonexclusive, royalty-free license to publish or reproduce the published form of this contribution, or to allow others to do so, for U.S. Government purposes. The Los Alamos National Laboratory requests that the publisher identify this anticle as work performed under the auspices of the U.S. Department of Energy. 


\title{
ORIENTATION IMAGING MICROSCOPY: NEW POSSIBILITIES FOR MICROSTRUCTURAL INVESTIGATIONS USING AUTOMATED BKD ANALYSIS
}

\author{
Brent L. Adams ${ }^{\vartheta}$, David J. Dingley§, Karsten Kunze ${ }^{\searrow}$, and Stuart I. Wright ${ }^{\dagger}$ \\ $\checkmark$ Department of Manufacturing Engineering and Engineering Technology \\ Brigham Young University, Provo, UT 84602 (USA) \\ $\S$ Department of Physics, Bristol University, Bristol, UK \\ †Los Alamos National Laboratory, Los Alamos, NM 87545 (USA)
}

Keywords: Microdiffraction, Backscattered Kikuchi Diffraction, Microtexture.

\begin{abstract}
A new microscopy, called Orientation Imaging Microscopy, is described. Imaging results from precise measurements of local lattice orientation rapidly obtained by Backscattered Kikuchi Diffraction. The hardware configuration of the microscope is described, and a description of image formation is presented. Applications of the method to several materials of differing lattice structure are described. Connections of the microscopy with various aspects of modem texture analysis are emphasized.
\end{abstract}

\section{INTRODUCTION}

This paper describes a new microscopy for probing microstructure in polycrystalline materials. Polycrystalline microstructures are inherently stochastic in character, and therefore extremely rich in diversity; no single type of microscopy can resolve the entire spectrum of structures present. New forms of microscopy help discover new characteristics of microstructure which may be linked to properties of interest.

The microscopy described here is called 'Orientation Imaging Microscopy (OIM)' owing to the fact that contrast is formed from measured gradients of local lattice orientation. More specifically, Backscattered Kikuchi Diffraction patterns are analyzed to determine lattice orientation in small localized regions. In its current configuration, which is described later in the article, OIM utilizes a standard scanning electron microscope, operated in stationary beam mode, in conjunction with highgain television technology to interrogate the flux of backscattered electrons emanating from crystalline regions of size $\approx 0.2 \mu \mathrm{m}$. Recently developed algorithms for rapidly indexing the patterns, coupled with stage motion automation, enable scanning of the polycrystalline surface with a probe which is extremely sensitive to the precise lattice orientation at each scan point. The contrast obtained in such scanning results from precisely defined spatial gradients in lattice orientation present in the scanned surface.

At its current state of development it is evident that OIM offers a very attractive coupling of several disparate methods of microstructural inquiry. Standard optical or scanning electron microscopy relies heavily upon various mechanisms of contrast formation which are related to lattice orientation gradients, but in rather imprecise ways. For example, the effect of chemical etching of metallographic surfaces is the formation of a surface topography which is dependent upon crystal orientation. The disturbed layer between grains may be attacked at a different rate from the grain interiors. Detailed knowledge of lattice orientation or disorientation effects on etching is rarely known, and thus the precise origins of contrast in conventional microscopy is not known. The size and shapes of grains or crystallites, and their chemical phase is revealed, but it is not generally possible to discern the lattice orientation of individual components. In contrast, the standard methods of modern texture analysis, 
which rely upon x-ray or neutron diffraction, provide information about volume fractions of grains of specified lattice orientation, but no information about grain size, shape morphology, or spatial distribution. OIM couples these two aspects of microstructure together in a manner which provides exciting new insights.

We first describe the configuration of the hardware. Then, OIM is defined as a sequence of mappings which reveal contrast in gray scale or color based upon precise knowledge of lattice orientation gradients in the polycrystal. Examples of OIM for several materials and material conditions are used to illustrate the principal microstructural features recovered from the microscopy. Some connections to modern texture analysis are then described, including grain-boundary textures and higher-order textural information.

\section{THE METHOD OF BACKSCATTERED KIKUCHI DIFFRACTION}

When a focused beam of electrons enters a crystalline material a shower of backscattered electrons emanates in all directions within a small interaction volume (of diameter $\approx 0.2 \mu \mathrm{m}$ at $20 \mathrm{KeV}$ ). The energy of a small fraction of these electrons is reduced only slightly from their initial value, even though their momentum is dramatically altered. Some of these electrons backscatter through the surface, where the flux pattern they form clearly marks bands diffraction emanating from planes in the crystal lattice. The basic physics of backscattering diffraction is well described in an early paper of Blackham, Alam and Pashley [1] which refers to the patterns as 'high-angle Kikuchi patterns.' Reference to 'high-angle' comes from the fact that optimal efficiency for the backscattering effect occurs when the outward sample normal is at large angles from the incident beam direction (typically 110 degrees). Although we shall refer to the patterns as Backscatter Kikuchi Diffraction patterns, or BKD patterns, the terminology Electron Back Scattered diffraction Patterns, or EBSP's, is also currently in widespread use.

BKD patterns are believed to contain a wealth of information about the local structure of the material, but only the lattice orientation will be of interest in this paper. By lattice orientation we refer to the rotation required to bring a reference lattice into coincidence with the local lattice, ignoring any small (elastic) distortions that may be present. We shall refer to ' $\mathrm{g}$ ' as the lattice orientation. It is known that $\mathrm{g}$ belongs to the 3 -dimensional orthogonal group $\mathrm{O}(3)$.

The embedding of a local frame in the lattice is typically not unique. Since physically distinctive orientations of the lattice are invariant under the action of elements of the point symmetry group (i.e. under different choices of the local lattice basis), we can restrict our attention to the homogeneous space of distinctive lattice orientations, $\mathrm{O}(3) / \Gamma$, which is the quotient space of right cosets of elements of $\mathrm{O}(3)$ with elements of the point symmetry group $\Gamma$. (For centro-symmetric lattices, the space of distinctive lattice orientations can be reduced to $\mathrm{SO}(3) / \Gamma$, where $\mathrm{SO}(3)$ is the 3-dimensional 'special' orthogonal group of rigid body rotations.)

The reader will also note that $g=g(x)$ in polycrystals. Since we shall be interested in gradients of $\mathbf{g}(\mathbf{x})$, we are interested, not only in $O(3) / \Gamma$, but also in the subspace of $O(3) / \Gamma$ which contains all physically distinctive disorientations of the lattice. Let $g$ and $g^{\prime}$ denote two orientations in $O(3) / \Gamma$. The disorientation between $\mathrm{g}$ and $\mathrm{g}^{\prime}, \Delta \mathrm{g}$, is defined by $\mathrm{g}^{\prime}=\Delta \mathrm{g} \cdot \mathrm{g}$. The subspace of physically distinctive disorientations, $\Gamma / O(3) / \Gamma$, is defined as the quotient space of left and right cosets of $O(3)$ with $\Gamma$.

Using an electron-sensitive photographic plate positioned inside the diffraction chamber, Venables and Harland reported obtaining high quality BKD patterns in 1973 [2]. In 1986, more than a decade later, Dingley and Baba-Kishi described a system for interrogating BKD patterns, formed on a phosphor screen located inside the microscope chamber, with a high-gain television camera located outside the chamber [3]. This novel advance led to interactive computer systems which allowed the operator to rapidly index BKD patterns for lattice orientation [4-6]. In this mode the operator is required to identify two or more known crystallographic zone axes in the pattern. From this information the lattice orientation is easily computed. Another version of this mode requires the operator to identify several diffraction bands, from which the interplanar angles and band widths are obtained in order to index the pattern for lattice orientation. When implemented on a personal 
computer, a skilled operator can index about 100 patterns per hour. The process is very fatiguing, however, and therefore rather prone to indexing errors when large data sets are needed.

When indexing is required from a very large number of locations in the sample (i.e.. from many locations on the section plane exposing the microstructure) manual indexing is rather impractical. Since 1990 three methods have been explored which isolate individual diffraction bands in the BKD patterns. Juul-Jensen and Schmidt detected bands with an algorithm which scanned the BKD image line-by-line, finding the local intensity maxima[7]. A scheme was developed whereby these local maxima could be connected together to identify the positions of bands. Wright and Adams [8] developed a scheme in which the 'Burns' algorithm [9] is used to extract edges from the local intensity gradients present in the patterns. It was shown that by examining long distance spatial correlations of these gradients over the pattern, lines of correlation are obtained which coincide with the edges of the bands, thereby enabling an indexing procedure. More recently, the identification of peaks in the Hough transform (which correspond to lines in the BKD pattern) has been employed by Lassen [10] and Kunze[11] and their co-workers to find bands.

As determined by careful comparisons, indexing via the Burns and Hough algorithms seems to be equally robust and reliable, although each method has advantages for certain materials and material conditions [i1]. In the system described in this paper, the indexing of a single BKD pattern requires, typically, 1-1.6 seconds of computer time, depending upon the crystal lattice. This rate is also quite sensitive to the quality of the images, which affects the number of television frames which must be averaged to reduce noise in the patterns. For very high quality patterns, where averaging is not an issue, the rate is also limited by the speed of the algorithm. The reliability and precision of the Burns and Hough approaches to automated indexing have been studied and reported [11]. Image quality parameters have been developed for each algorithm, and indexing reliability is found to be a strong function of image quality. When BKD patterns of low image quality are eliminated from consideration. the reliability of correct indexing typically exceeds $99 \%$ for both algorithms. If lower quality patterns are admitted, then this number will decrease. The angular precision with which BKD patterns can be indexed is more difficult to ascertain. Comparison of automated indexing results with very carefully (manually) indexed sets has shown that the typical mean disorientation between the automated results and the manual results is less than 1 degree; this result is at least equivalent, and arguably better, than similar comparisons between sets which were manually indexed by more than one operator.

Test calculations[12] have also demonstrated that the established algorithms are able to correctly index simulated patterns from any possible orientation in the fcc and bcc crystal structures. In other words, it has not been possible to detect any 'dark corners' where the indexing algorithms are not able to correctly index the simulated patterns. Even with the inclusion of simulated experimental errors in the considered band parameters, the indexing procedures remain stable. (We point out that non-unique solutions can occur in cases where an insufficient number of bands has been detected. This happens with patterns of low band contrast as well as when the solid angle in the interrogated diffraction cone is not sufficiently large.)

Based upon these results we have concluded that automated indexing of BKD patterns results in an improvement over manual indexing, in both reliability and precision.

\section{ORIENTATION IMAGING MICROSCOPY}

Although the concept of orientation imaging was suggested at least two decades ago by Haessner and coworkers[13], the potential of orientation scanning as a new form of microscopy could not be realized until rapid, automated indexing of diffraction patterns became a reality. When rapid indexing is coupled with computer controlled stage (or beam) motion, Orientation Imaging Microscopy is realized.

The current hardware configuration for OIM is now described. High quality BKD patterns are obtained using an silicon integrated target (SIT) fiber optic camera. The fiber optic bundle is coated on one end with a phosphor. Use of a fiber optic removes image distortions present in conventional camera lenses. The camera control unit is capable of averaging a variable number of video images (up to 128); the actual number used is based upon the typical quality of the patterns, and is a function of Znumber, strain level in the sample, and the quality of the mechanical and electro-polishing procedure. A background image is obtained from a large number of grains when the SEM is in scanning mode. This background image is stored, and then subtracted from each averaged image. The sample holder is 
fixed upon a piezoelectric $x-y$ stage, which is mounted such that the outward sample normal makes an angle of 110 degrees from the direction of the electron beam. The flat sample surface lies in the eucentric plane such that its surface remains in focus with motions of the $x-y$ stage. The stage is capable of motions as small as $0.1 \mu \mathrm{m}$, with a total range of travel of $27 \mathrm{~mm} \times 26 \mathrm{~mm}$. Reproducibility over the entire range of travel is $0.25 \mu \mathrm{m}$. The camera control unit and the stage control unit are interfaced to a workstation with 85 mips performance. This computer provides central control for both the stage and the video camera unit. A frame capture board is installed in this computer to facilitate capture of the BKD patterns from the camera control unit. Algorithms for indexing the patterns require $\approx 1.2$ seconds in the case of cubic and $\approx 1.6$ seconds for trigonal lattices.

We have formally defined OIM in terms of a sequence of three mappings [14]. Here, however, we shall use a more informal description which carries the essence of the full definition. Local orientation measurements are obtained on a regular array of points positioned on flat polished sections. A representation of this array of points, appropriately scaled, is also introduced into the computer graphics system. Overlapping the representation, the investigator is able to introduce various graphical units, including pixels differentiated by color or gray scale. Color and gray scale are assigned on the basis of local details of the lattice orientation. These graphical units are used to form a visualization of some aspect of the microstructure.

A simple example may help to illustrate the process. The investigator may be interested in highlighting large-angle grain boundaries in the material. A measurement array must be selected such that the scale of the array is much smaller than the grain-size of the material. (Obviously, the graphical representation of this array is typically scaled or magnified to a much larger dimension.) If the absolute minimum disorientation between the lattice orientation of neighboring points in the measurement array, say

$$
\left.\omega\left(g, g^{\prime}\right)=\operatorname{arcos}\left\{\frac{1}{2}\left(\max _{j=1 \ldots j} \operatorname{trace}\left(\gamma_{j} g^{\prime} g^{-1}\right)-1\right)\right)\right]
$$

exceeds 15 degrees, then a line segment is introduced in the graphics system. For a square array of measurement points this line segment would be of length equal to the dimension of the array, and positioned with its midpoint lying midway between the selected points, and perpendicular to their connecting line. When all possible pairs or points in the array are considered, the graphical system will show a connected set of lines representing boundaries of large disorientation. This representation of boundaries constitutes one form of an Orientation Imaging Micrograph.

Other features of microstructure are formed in analogous fashion. For example, colored pixels can be used to identify grains with lattice orientations of interest to the investigator. Line segments can be altered in thickness or gray scale to signify disorientations with special meaning, such as those belonging to coincident site lattices. It has also proven to be of great help to use gray scale as a means of identifying the quality of BKD patterns. Thus, regions of higher strain or altered chemical composition are highlighted. The number of possible ways to form OIM's is unlimited. Several examples are provided in the next section.

\section{EXAMPLES OF ORIENTATION IMAGING MICROGRAPHS}

A simple example of an orientation imaging micrograph is given in figure 1. Approximately fourteen thousand measurements were made on calcite. The measurements were made on a regular hexagonal grid with $5 \mu \mathrm{m}$ between each measurement point. The grain boundary map was reconstructed from the measurements by drawing a thin line between neighboring measurement points when the misorientation exceeds $5^{\circ}$ and thick line when the misorientation exceeds $15^{\circ}$. Clearly, this method delineates the grains quite well. In addition, some indication of the character of the boundaries is retained in this figure. 


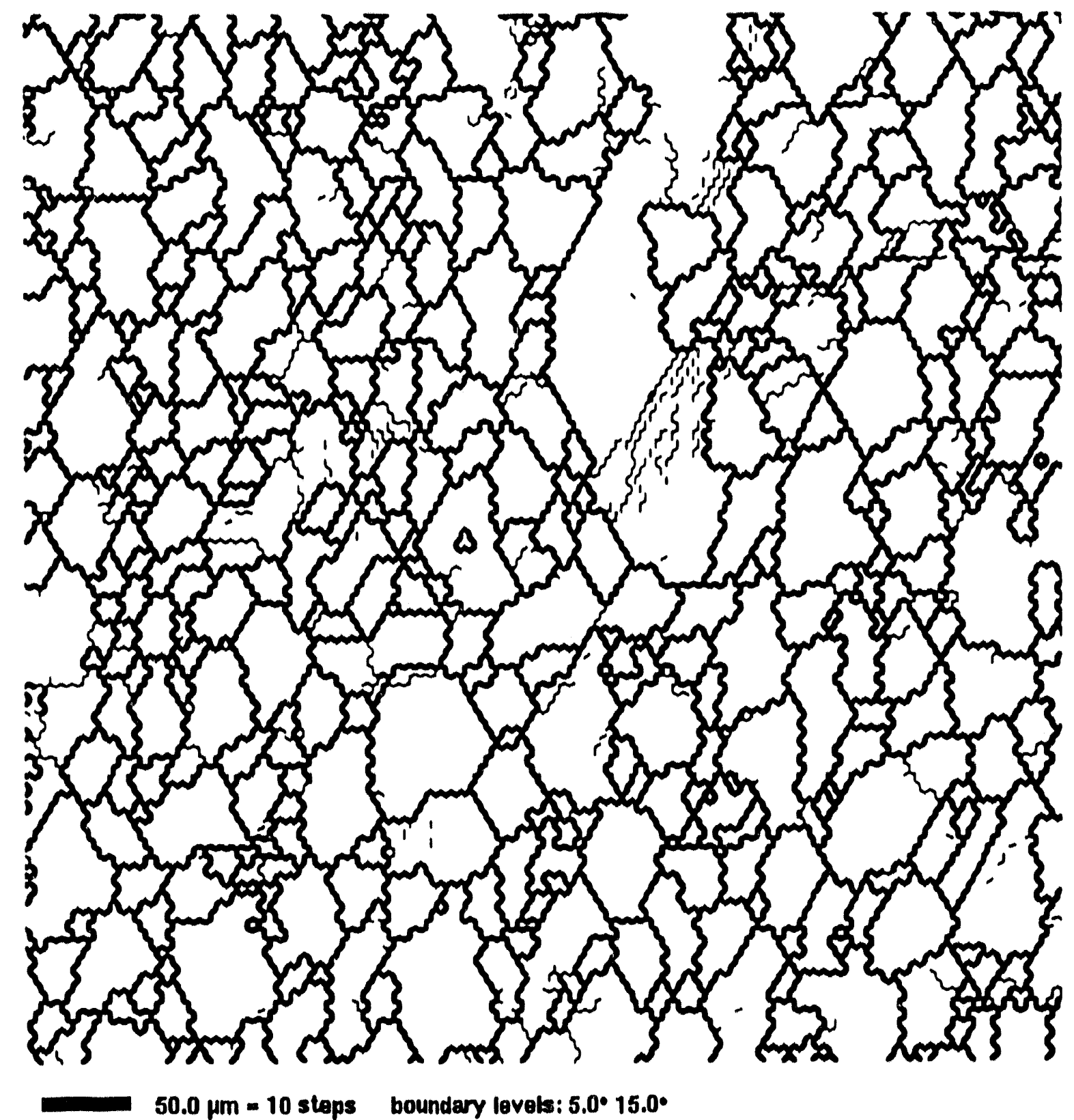

Figure 1 Grain boundary map reconstructed from automatic orientation measurements on calcite.

The character of the grain boundaries can be captured in the orientation imaging micrograph by highlighting boundaries of a special character. Figure 2 shows an image constructed from automatic orientation measurements on OFE half hard copper recrystallized at $600^{\circ} \mathrm{C}$ for 1.5 hours. The orientation measurements were made on a regular hexagonal grid with $20 \mu \mathrm{m}$ spacing between measurement points. The grain boundary map was reconstructed by drawing lines between neighboring measurement points when the misorientation angle exceeds $15^{\circ}$. In addition $\Sigma 3$ and $\Sigma 9$ boundaries are highlighted. $\Sigma 3$ bound :es are double thickness and $\Sigma 9$ boundaries are triple thickness. In this sample the $\sum 3$ boundaries occur at 15 times random while $\sum 9$ boundaries occur at 12 times random. If color is used in the representation, then more types of boundaries can be effectively visualized. 


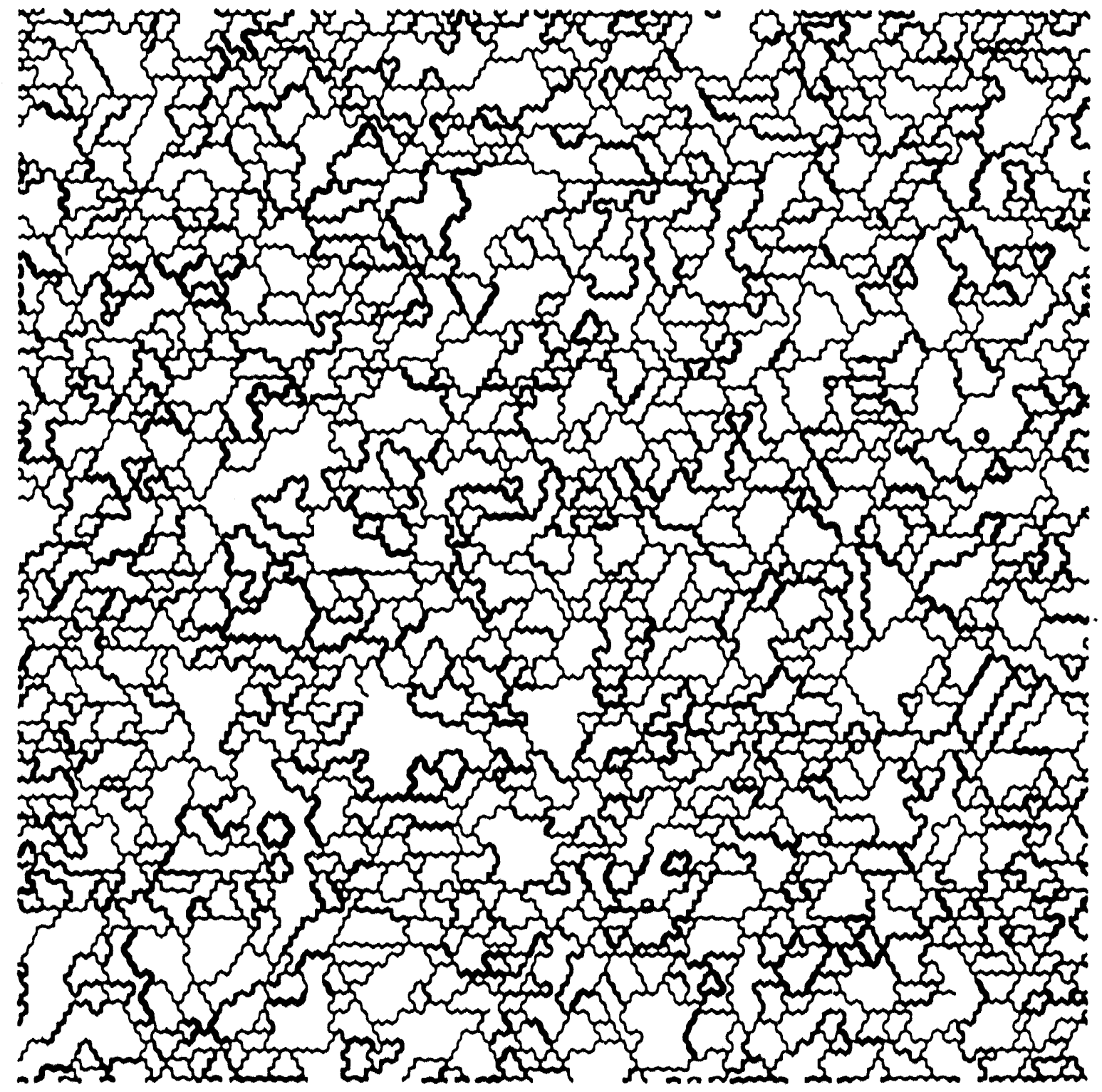

$200.0 \mu \mathrm{m}=10$ steps boundary levels: $15.0^{\circ}$

Figure 2 Grain boundary map reconstructred from automatic orientation measurements on OFE copper. $\Sigma 3$ boundaries are denoted by double thick lines and $\Sigma 9$ boundaries by triple thick lines.

In addition to the micrograph type images formed from the discrete measurements of orientation, the data holds a wealth of statistical information. For example, from the measurements made on the recrystallized OFE half hard copper, the misorientation was calculated between orientations separated by one, three and seven average grain diameters in the rolling direction. The relative frequency of occurrence of the misorientation between these neighbors versus misorientation angle is plotted in figure 3. The three curves essentially represent the distribution in misorientation angle of first, third and seventh nearest neighbors. The seventh average grain diameter curve is essentially the random misorientation distribution curve. Misorientations below $15^{\circ}$ were omitted in order to not skew the scaling of the graph. 


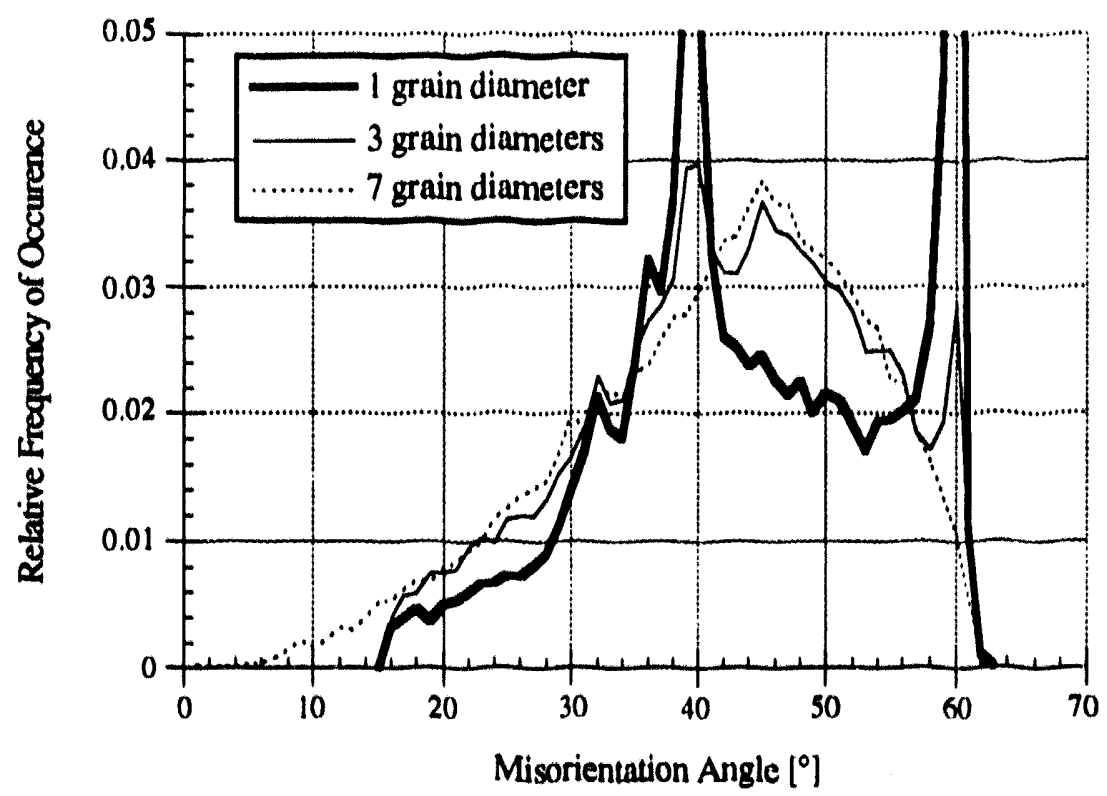

Figure 3 Relative frequency of occurrence versus misorientation angles

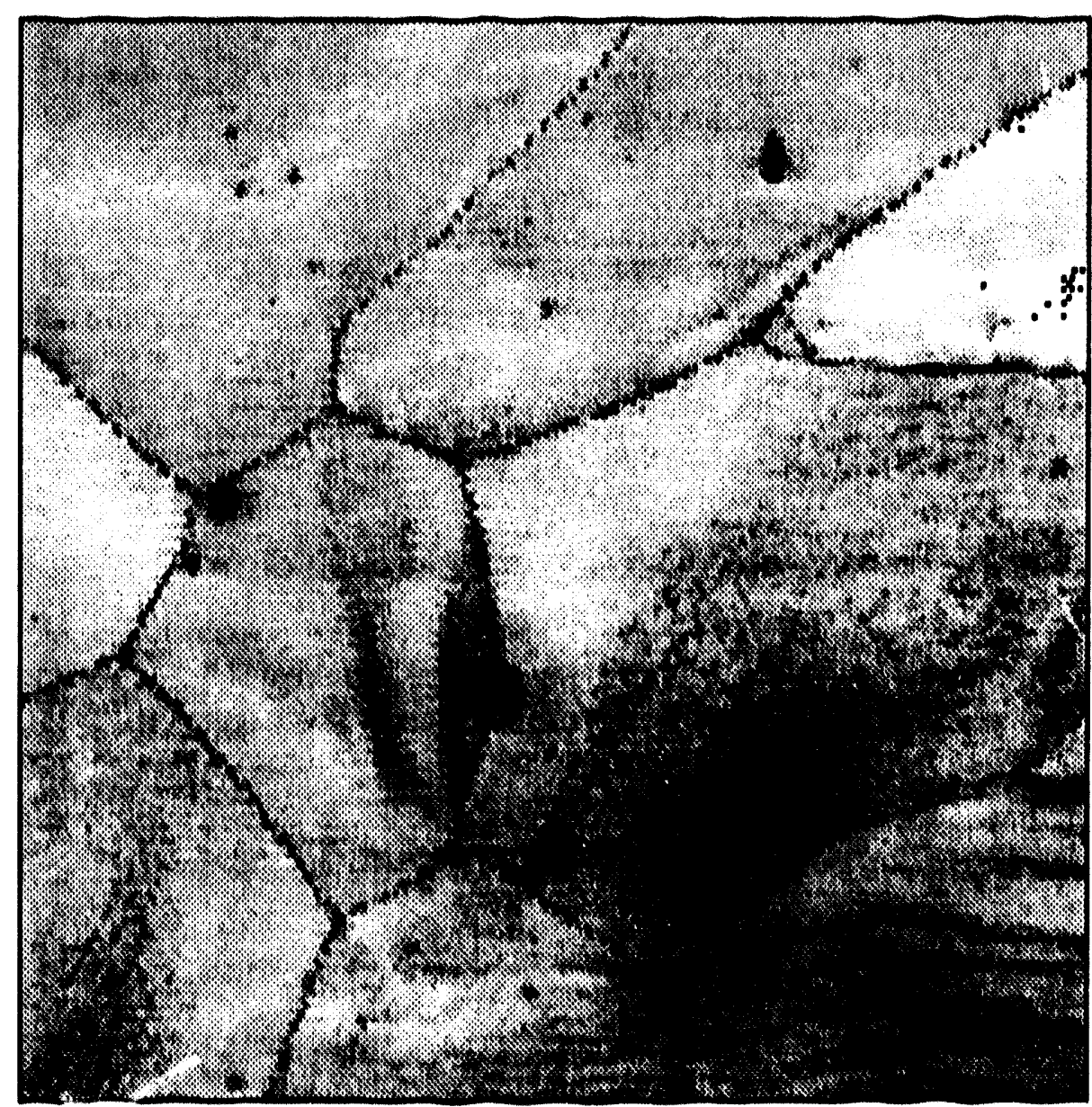

Figure 4 Image formed by differences in BKD pattern quality on phosphor doped iron-3\% silicon. 
Another example of an image that can be formed from the automatic orientation measurements is an image formed by considering the quality of the diffraction patterns associated with each measurement. A parameter describing the BKD pattem quality can be mapped onto a gray scale to create contrast in an image. Points having higher quality diffraction patterns associated with them will appear lighter than points having lower quality patterns. Using this technique, an image (figure 4) was formed from measurements on rolled iron-3\% silicon doped with $200 \mathrm{ppm}$ phosphorous. In this sample, stress was developed by a microhardness indent just outside the bottom right hand comer of the image. The small dark spots are points from which an orientation could not be determined. The darker blotches are due to either precipitates or to defects on the specimen surface such as pits or debris. It is interesting to note that significant variations in image contrast occur within the individual grain interiors. The darker regions likely represent areas of relatively high dislocation density.

The spread of orientation within grain interiors can be visualized using the measurements. In figure 5, the deviation (up to $20^{\circ}$ ) from a reference orientation has been mapped onto a gray scale. The reference orientation was selected by locating the peak in an ODF calculated from only those measurements which lie within the grain. The isolated grain in this figure is the grain just left of center in figure 4.

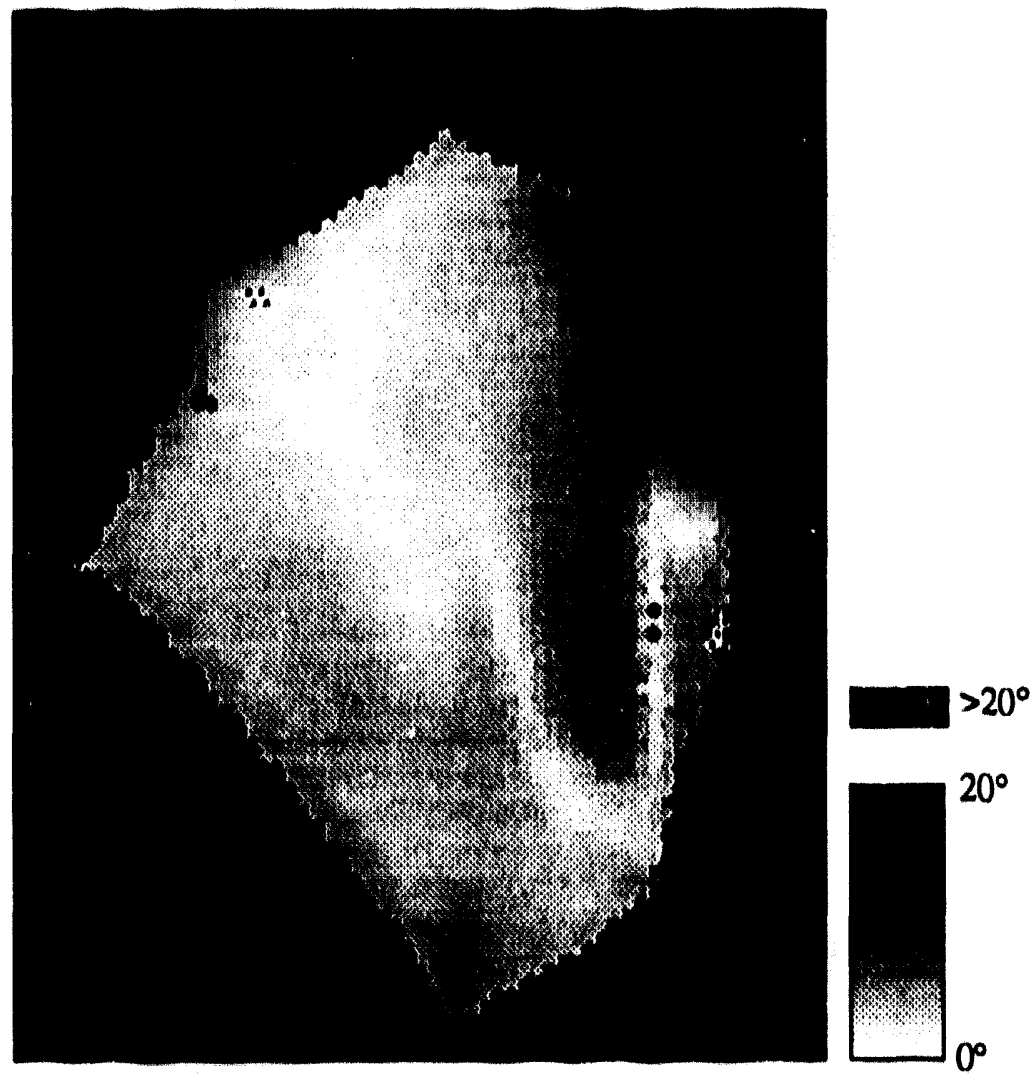

Figure 5 Deviation from an ideal orientation within a single grain represented with gray levels for a grain in figure 4.

The automatic technique is also extremely valuable for exploring local orientation relationships and the spatial distribution of microtexture in heterogeneous microstructures. One such example is the affected zone near an electron beam weld in beryllium. Figure 6 shows an orientation imaging micrograph from the beryllium. Some of the aspects of this material that are currently being explored are the preferred crystallographic growth direction, which crystal planes lie preferentially parallel to the weld and the microtexture and misorientation texture in the affected and unaffected zones. 


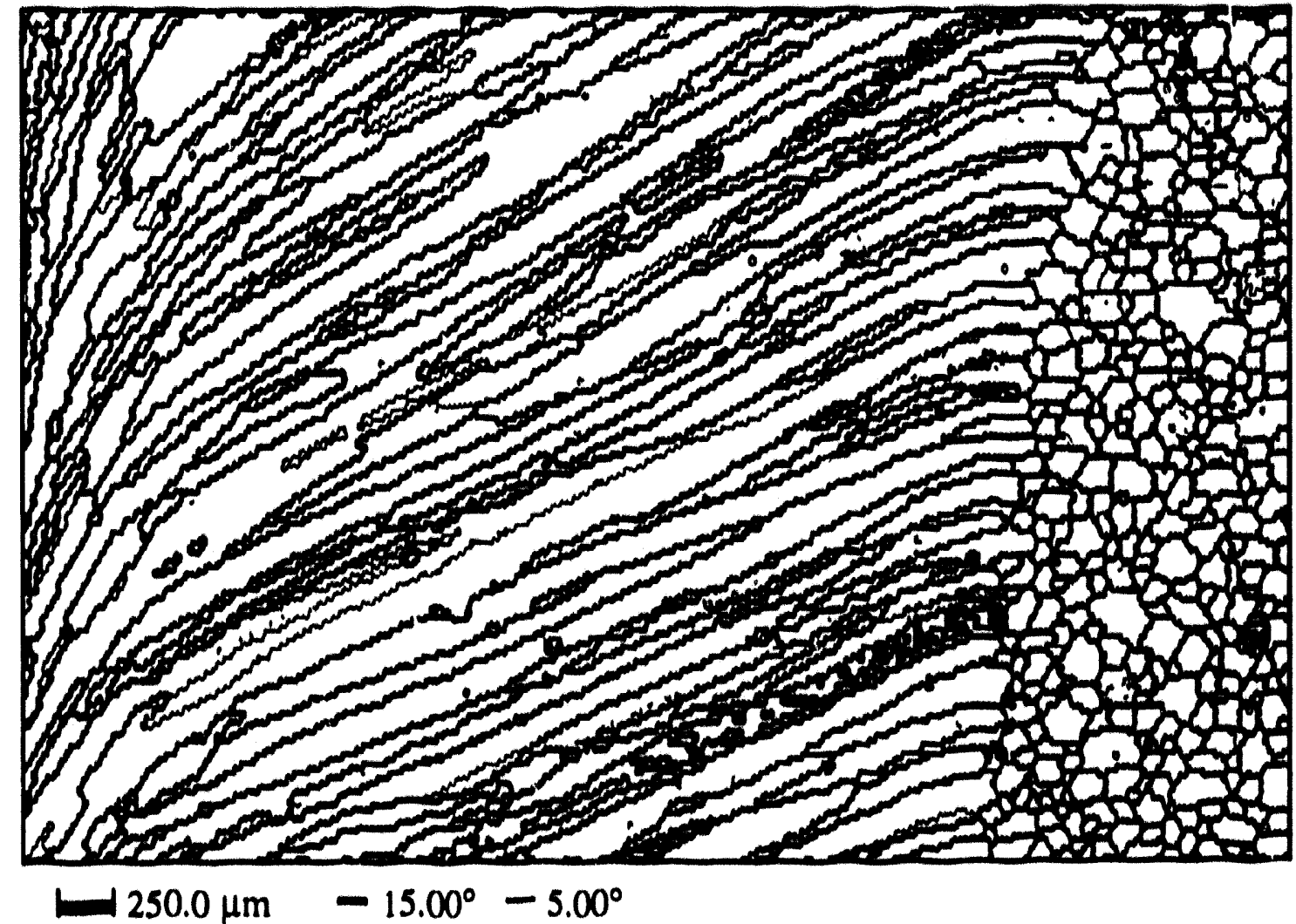

Figure 6 Orientation imaging micrograph from an electron beam weld in beryllium.

A less glamorous example of the utility of OIM is dealing with small or unusually shaped samples where it may be difficult to prepare adequate surfaces for $x$-ray diffraction. For example, the texture of a small tube formed by chemical vapor deposition (CVD) of tantalum was measured by $x$-ray diffraction and by OIM. The orientation imaging micrograph is shown in figure 7 . The cluttered regions are due to deformation imposed on the sample at different stages in the CVD process.

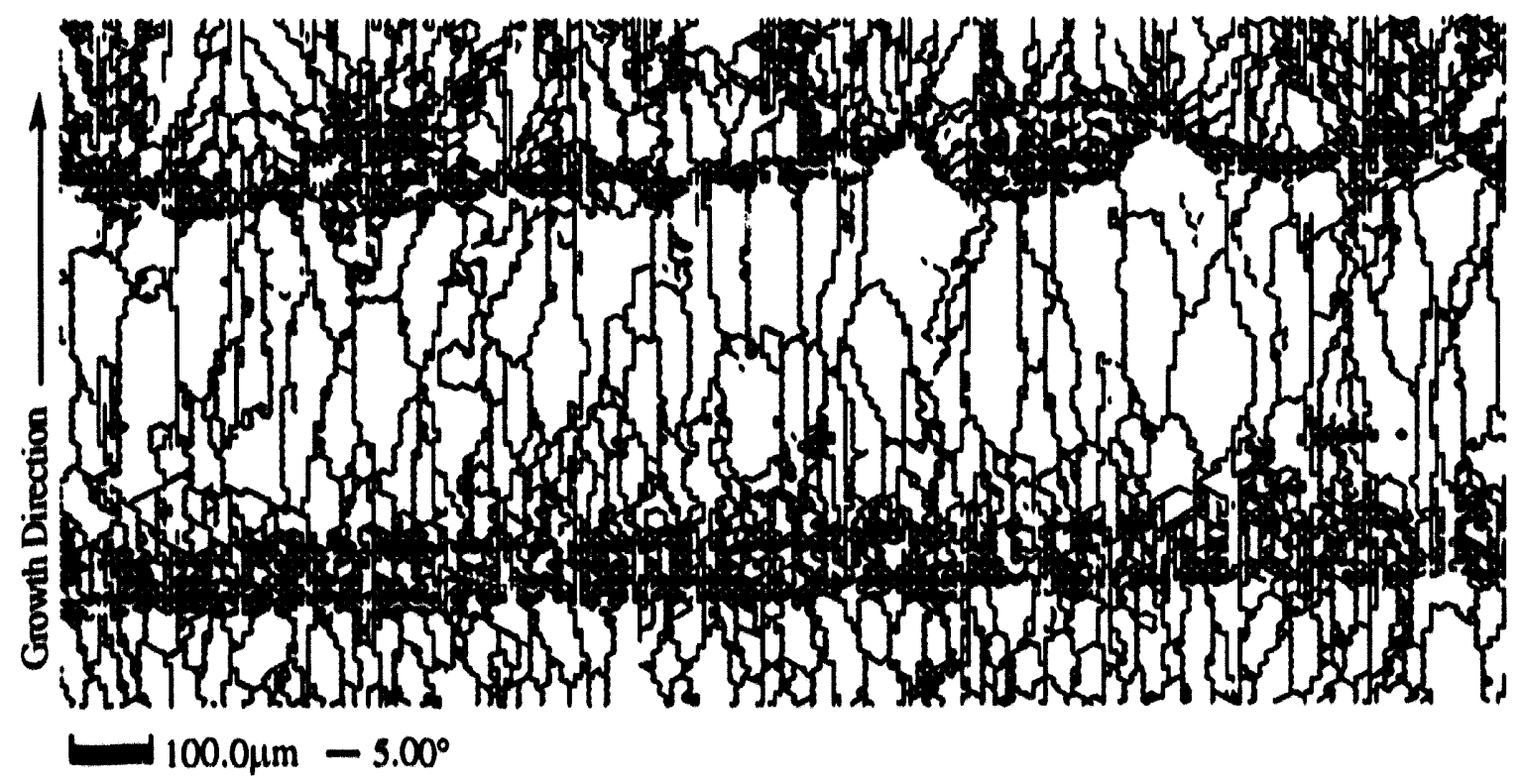

Figure 7 OIM from a tantalum tube formed by chemical vapor deposition. 
The texture determined by $x$-ray diffraction indicated a fairly random texture. However, OIM indicated a strong $(100)$ fiber texture. The difference in the textures arises from the preparation of the sumple for the texture measurements. In creating a flat surface for $x$-ray diffraction a spread in the $(100)$ fiber texture is introduced artificially as shown in figure 8.
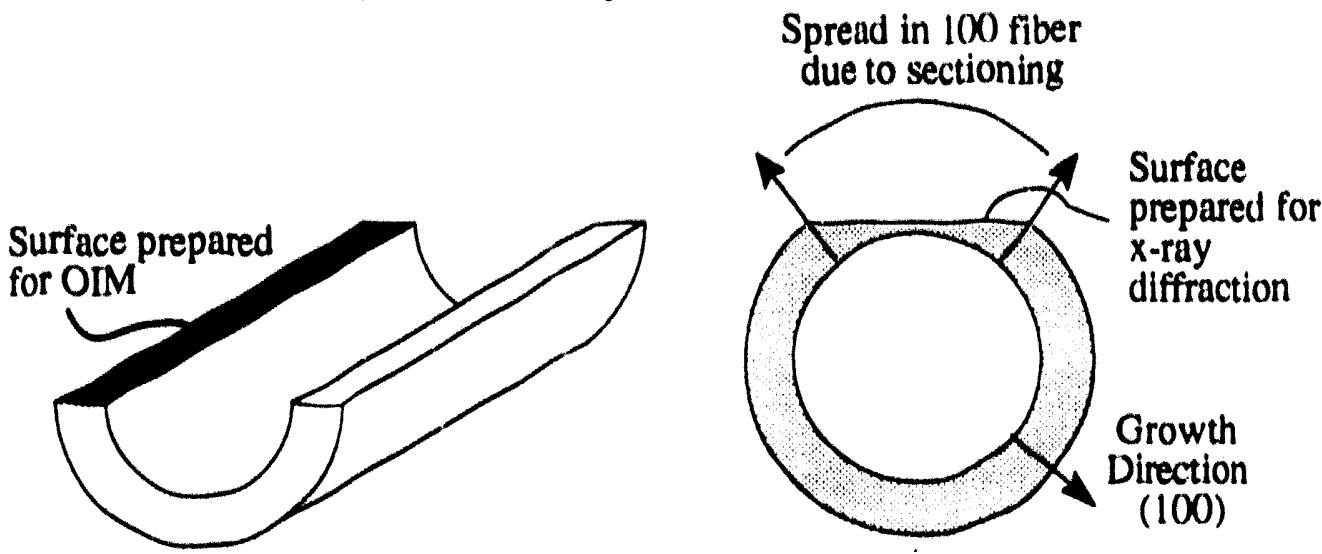

Figure 8 Schematic of texture measurement regions on a tantalum tube formed by chemical vapor deposition.

\section{THE CONNECTIONS BETWEEN MODERN TEXTURE ANALYSIS AND OIM}

It is instructive to consider the connections between crystallographic texture analysis and OIM. The principal object of interest in modem texture analysis is the orientation distribution function (odf). Formally, the odf is a function over the space of distinct orientations of the lattice to the positive real line: i.e., $\mathbf{f}: \mathbf{G} \rightarrow \mathbb{R}$, where $\mathbf{G}=O(3) / \Gamma$. It gives the volume density of occurrence of lattice orientation $g \in G$ in the polycrystal. Let $d g$ denote the invariant measure of an infinitesimal neighborhood, $N(g) \subset G$ surrounding $g$. (Invariant measures ensure that any choice of partitioning of $\mathbf{G}$ into neighborhoods always results in an identical integrated measure over the whole space $\mathbf{G}[15]$.) We choose the normalization of the invariant measure such that

$$
\int_{Q} d g=1
$$

The odf is then defined to be the volume density of the polycrystal associated with crystallites of orientation $g$; thus, for $g \in N(g)$ with invariant measure $d g$ we have

$$
d V / V=f(g) d g \text {. }
$$

So defined, the odf has an obvious normalization

$$
\int_{Q} f(g) d g=1
$$

The connection of the odf to OIM is straightforward. From Delesse's principle of stereology it is known that volume fractions can be equated to area fractions in random sections cut from the polycrystal. Thus if the area fraction $\mathrm{dA} / \mathrm{A}$ of crystallites exposed in the section plane with lattice orientation in the neighborhood $N(g)$ is known, then

$$
d A / A=f(g) d g \text {. }
$$

In the context of OIM, this area fraction can be equated to a number fraction of array points which fall in the neighborhood $N(g)$. Let this number fraction be $d N / N$; it follows that

$$
d N / N=d A / A=d V / V=f(g) d g \text {. }
$$


Of increasing interest to modern texture analysis is the study of grain-boundary texture. A principle motivation for the development of OIM has been the ability to directly measure grainboundary texture. We now describe certain connections between OIM and sstablished methods of stereology which have the object of discovering the surface area per-unit-volume of intercrystalline interfaces which exhibit specified physical orientation and lattice orientation on each side of the interface. Stereology defines relations connecting this function with the number of intersections of a random line per-unit-length of line with boundaries of particular character, or with the direct observation of interface intersections with the section plane. Here. a few details of these connections are stated, without proof. The interested reader will need to consult the work of Hilliard[16], Adams. [17] and Adams and co-workers [18-19] for technical details.

Let the function $S_{V}\left(g, n, g^{\prime}\right)$ represent the surface area per-unit-volume density of interface with interface normal $\mathbf{n}$ and described by lattice orientation $\mathbf{g}$ in the direction of the tail of $\mathbf{n}$ and lattice orientation $g^{\prime}$ in the direction of the head of $n$. Thus $S_{V}: \mathbf{G} \otimes S^{2} \otimes \mathbf{G} \rightarrow \mathbb{R}$ where $S^{2}$ denotes the unit sphere of possible directions of the interface normal vector $\mathbf{n}$. Our focus shall be upon a 'non-sided' form of this function defined by

$$
\tilde{S}_{V}\left(g, n, g^{\prime}\right)=\left[S V\left(g, n, g^{\prime}\right)+S_{V}\left(g,-n, g^{\prime}\right)\right] / 2 \text {. }
$$

Define the 2-point function $f_{2}(g, g \mid r), f_{2}:(\mathbf{G} \otimes \mathbf{G} \rightarrow \mathbb{R}$, to be the number fraction density of point-pairs on the measurement array, separated by vector $r$ which have orientation $g$ at the tail of $r$ and $g^{\prime}$ at it's head. Let $d N\left(g . g^{\prime} \mid r\right)$ be the number of pairs which have the tail falling in an infinitesimal neighborhood $\mathrm{N}(\mathrm{g}) \subset \mathrm{G}$ of measure $\mathrm{dg}$ surrounding $\mathrm{g}$, and their head falling in an infinitesimal neighborhood $\mathrm{N}\left(\mathrm{g}^{\prime}\right) \subset \mathbf{G}$ of measure $d \mathrm{~g}^{\prime}$ surrounding $\mathrm{g}^{\prime}$. This number is given by $f_{2}\left(g, g^{\prime} \mid \mathbf{r}\right)$ according to

$$
d N\left(g, g^{\prime} \mid r\right) / N=f_{2}\left(g, g^{\prime} \mid r\right) d g d g^{\prime}
$$

where $\mathrm{N}$ now represents the total number of point-pairs accessible in the measurement array.

In the limit that the magnitude of $\mathbf{r}$ is small compared to the characteristic size of the constituent crystallites present in the polycrystal. there exists a formal connection between the 'non-sided' average of $f_{2}\left(g, g^{\prime} \mid r\right)$, denoted as $\tilde{f}_{2}\left(g, g^{\prime} \mid r\right)=\left[f_{2}\left(g, g^{\prime} \mid r\right)+f_{2}\left(g, g^{\prime} \mid r\right)\right] / 2$, and $\left.\widetilde{S}_{V}\left(g, n, g^{\prime}\right) \mid 20\right)$. The fundamental stereological relation is

$$
\operatorname{limit}_{|\mathbf{r}| \rightarrow 0}\left[\tilde{f}_{2}\left(g, g^{\prime} \mid \mathbf{r}\right)=\int_{S^{2}} \tilde{S}_{V}\left(g, n, g^{\prime}\right)|\mathbf{r} \cdot \mathbf{n}| d \mathbf{n}\right]
$$

In this expression dn is just the measure of surface area for an infinitesimal patch of surface on $\mathrm{S}^{2}$ surrounding direction $\mathbf{n}$.

Although details cannot be provided here, this integral equation can be solved if sufficient measurements have been made in a sufficient number of directions of $r$ to adequately cover $S^{2}[18$. 20). The function $\widetilde{S} v\left(g, n, g^{\prime}\right)$ contains eight independent parameters (three for each of the orientation variables and two defining the direction of the interface normal). Often, it is of interest to reduce this function to simpler forms or projections which capture a restricted portion of the full information. Formally, this just requires integration of those variables to be eliminated over the full domain of their occurrence.

\section{SUMMARY AND DISCUSSION}

Orientation Imaging Microscopy presents views of polycrystalline microstructure of unprecedented detail. Because the observer is required to precisely define spatial and orientational resolution. OIM has a distinctively quantitative character as compared to ordinary forms of optical and scanning electron microscopy. We have illustrated this quantitative character by outlining connections of OIM with modern texture analysis, including stereological analysis of grain boundary textures. 
OIM will challenge some long-standing views in the materials science commurity. For example, it is no longer clear what is meant by grain size. Using conventional microscopy it is not easy to identify the character of boundaries from which the observed contrast originates. Any particular technique no doubt excluded certain boundaries, but the character of those excluded is not typically defined. With OIM all boundaries exhibiting absolute disorientations exceeding about one-half degree can be reliably imaged. The choice of parameters used to form the image will substantially influence the apparent grain size. (We emphasize that minimum disorientation is only one way of forming contrast by orientation imaging. Other choices might emphasize particular axes of rotation, or particular CSL character.) This leads us to wonder about the influence of grain boundary structure distribution on yield strength and other properties.

It is also evident that polycrystals show variations of disorientation on a single facet of the interface. These gradients are observed in fully recrystallized microstructures, and seem to increase in frequency and strength with deformation. It is the impression of the authors that the influence of this aspect of microstructure on observed phenomena and properties has not been extensively investigated.

Anothur interesting aspect of microstructures which can be studied via OIM is the connectivity of microstructure. Connectivities of the grain interior and interface components can be studied. It is suspected that such connectivity in microstructures strongly influences some properties of interest such as fracture, fatigue and some forms of corrosion.

\section{ACKNOWLEDGMENT}

The authors acknowledge sponsorship of this work by the National Science Foundation under a Materials Research Groups Award. The connections of this work to stereology were developed with funding from the Office of Basic Energy Sciences of the U.S. Department of Energy. The contributions of T. Mason, D. West, and Y. Pan of Brigham Young University for help with preparing the manuscript are acknowledged. Thanks to J. D. Cotton of Los Alamos National Laboratory and A. J. Sherman of Ultramet for providing some of the materials used in the examples.

\section{REFERENCES}

1) Blackham, M. N., M. Alam and D. W. Pashley: Proc. Royal Society of London, 1953, A221, 224.

2) Venables, J. A. and C. J. Harland: Philosophical Magazine, 1973, 27, 1193.

3) Dingley, D. J. and K. Baba-Kishi: Scanning Electron Microscopy, 1986, i․, 383.

4) Dingley, D. J.: Backscattering in the Scanning Electron Microscope, Institute of Physics Conference Series, 1990. No. 119, 551.

5) Dingley, D. J., A. Day, and A. Bewick: Proc. Ninth Int. Conf. Textures of Materials, eds. R. Pennelle and C. Esling,Textures and Microstructures, 1991, 14-18, 91.

6) Schmidt, N.-H., and N. Ø. Olesen: Canadian Mineralogist, 1989, $27,15$.

7) Juul-Jensen, D. and N.-H. Schmidt: Recrystallization '90, ed. T. Chandra. TMS, Warrendale, PA, $1991,219$.

8) Wright, S. I. and B. L. Adams: Metallurgical Transactions, 1992, 23A, 759.

9) Burns, J. B., A. R. Hanson, and E. M. Riseman: IEEE Trans. PAMI, 1986, 8, 425.

10) Lassen, N. C., K. Conradsen and D. Juul-Jensen: Scanning Microscopy International, 1992, $\underline{6}, 115$.

11) Kunze, K., S. I. Wright, B. L. Adams and D. J. Dingley: Textures and Microstructures, 1993, 20, 41.

12) Kunze, K.: unpublished research.

13) Gothardt. R., G. Hoschek, O. Reimold and F. Haessner: Texture, 1972, 1, 99.

14) Adams, B. L., S. I. Wright and K. Kunze: Metallurgical Transactions, 1993,24A, 819.

15) Gel'fand, I. M., R. A. Minlos and Z. Ya. Shapiro: Representations of the rotation and Lorentz groups and their applications, Pergamon Press, Oxford, 1963, 6.

16) Hilliard, J. E.: Trans. Am. Inst. Min. Engrs, 1962, 224, 1201.

17) Adams, B. L. : Metallurgical Transactions, 1986, 17A, 2199.

18) Adams, F. L. and D. P. Field: Metallurgical Transaciions, 1992, 23A, 2501.

19) Field, D. P. and B. L. Adams: Metallurgical Transactions, 1992, 23A, 2515.

20) Adams, B. L.: Materials Science and Engineering, 1993, A166, 59. 

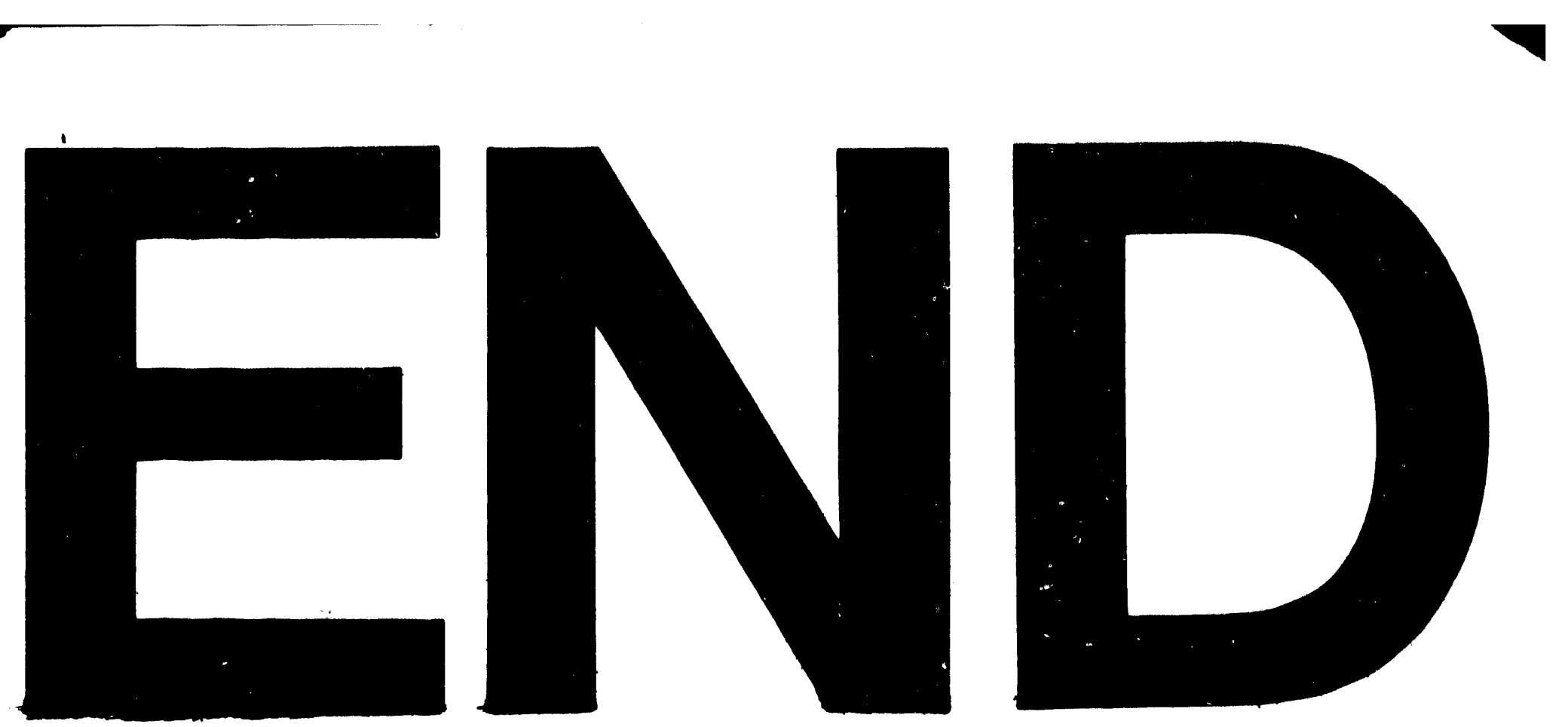

IIII-
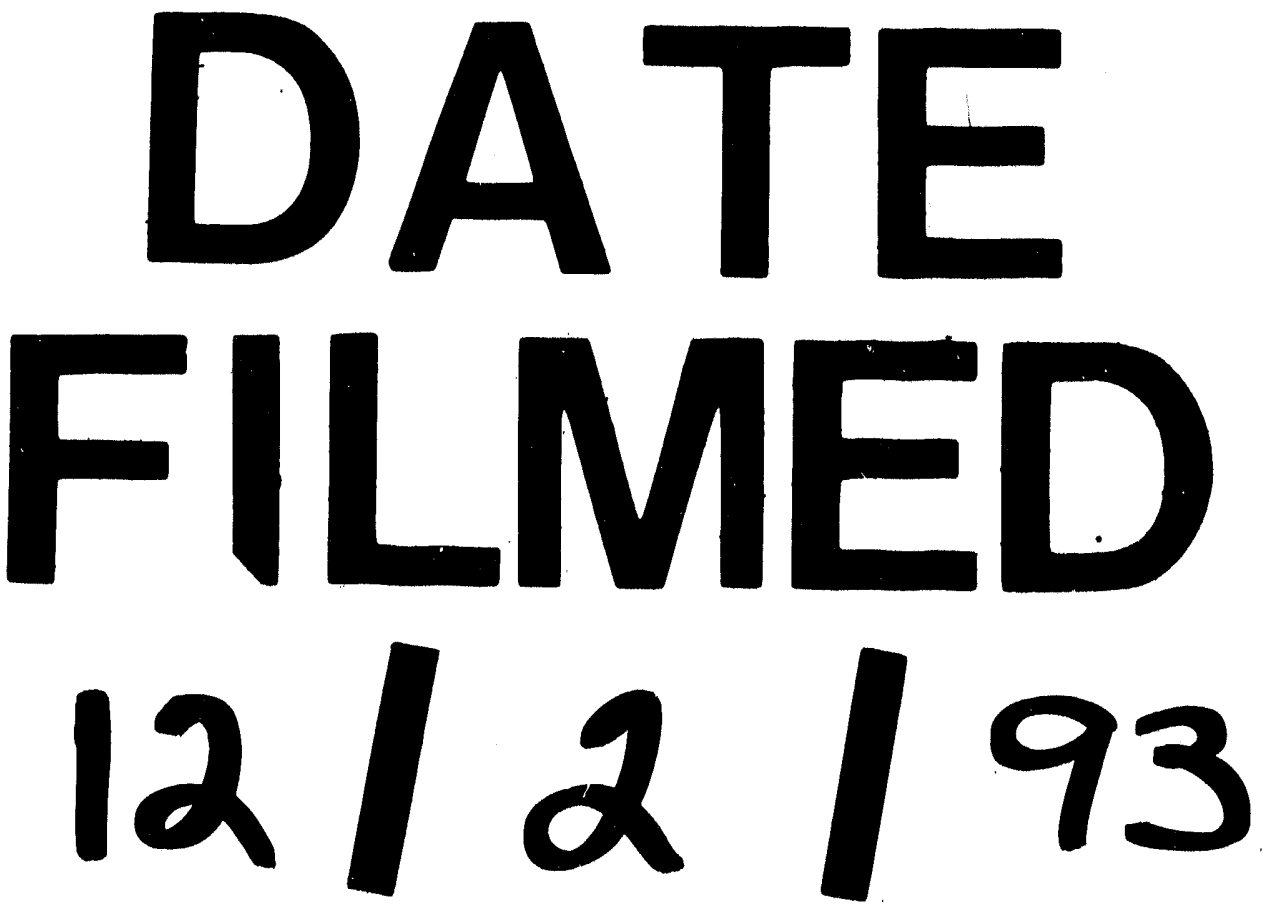


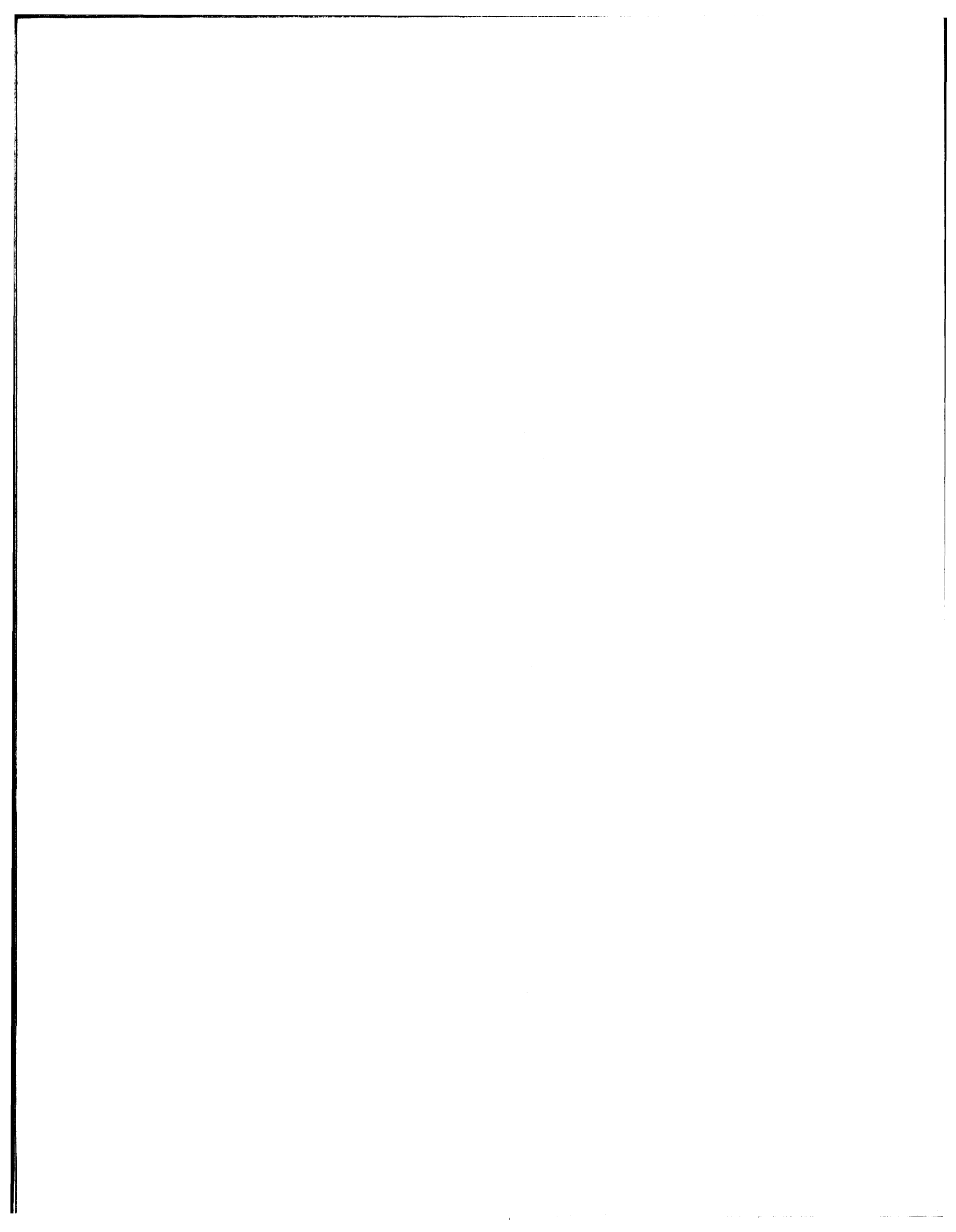

